\title{
THE EFFECT OF CALCIUM CARBONATE, ALUMINUM PHOSPHATE, AND ALUMINUM HYDROXIDE ON MINERAL EXCRETION IN MAN ${ }^{1}$
}

\author{
BY JOSEPH B. KIRSNER \\ (From the Frank Billings Medical Clinic, Department of Medicine, \\ University of Chicago, Chicago)
}

(Received for publication July 3, 1942)

\section{INTRODUCTION}

In order to elucidate further the mechanism of the alkalosis observed during the alkali treatment of peptic ulcer, a detailed study of mineral excretion following the administration of relatively nonabsorbable antacids seemed desirable. Hence, an investigation of the influence of calcium carbonate on mineral excretion in man was undertaken, and its action compared with the effects of aluminum hydroxide and aluminum phosphate, two nonabsorbable compounds.

\section{METHOD OF STUDY}

Studies were carried out in 2 adult men and one female with peptic ulcer, in all of whom previous observations had indicated renal function to be satisfactory. A special metabolic ward and nursing staff were utilized. The patients were maintained on special ulcer diets (Tables I and II), without change, during the entire experiment. Chloride-free distilled water was used for drinking purposes. The daily fluid intake averaged approximately

TABLE I

Composition of diet employed in case $A . K$. (Daily values)

\begin{tabular}{l|c|c}
\hline \hline & $\begin{array}{c}\text { Special } \\
\text { diet }\end{array}$ & $\begin{array}{c}\text { Standard values } \\
\text { for sedentary } \\
\text { adult female }\end{array}$ \\
\hline Carbohydrates & 198 & \\
Protein & 72 & \\
Fat & 104 & \\
Calories & 2016 & \\
Chloride (mgm.) & 2974 & \\
Sodium (mgm.) & 2029 & 3052 \\
Potassium (mgm.) & 3052 & 1320 \\
Calcium (mgm.) & 1159 & 12 \\
Phosphate (mgm.) & 1342 & 5000 \\
Iron (mgm.) & 10.5 & 1.2 \\
Vitamin A (I.U.) & 9570 & 1.8 \\
Thiamin (mgm.) & 1.014 & 70 \\
Riboflavin (mgm.) & 2.51 & \\
Ascorbic Acid (mgm.) & 78 & \\
Vitamin D (I.U.) & 38 & \\
& & \\
\hline
\end{tabular}

1 This work was supported in part by a grant from John Wyeth and Bro., Inc., Philadelphia, $\mathrm{Pa}$.
3000 cc. The daily urinary output varied from 1200 to $3000 \mathrm{cc}$. There was no significant change in body weight during the entire experiment. The only medication permitted, aside from the substances studied, was the occasional administration of cascara to facilitate daily bowel activity, ${ }^{2}$ and the use of mild sedatives for sleep. An interval of 4 to 6 days was allowed for adjustment to the diet, prior to the control observation. Mineral excretion was studied over periods of 4 days duration each. Ten grains of carmine were given at the onset of each period and repeated 96 hours later; the feces obtained between the carmine markers was collected for analysis. The 4-day collection was mixed thoroughly; accurately weighed 2.0 gram samples were taken for the various determinations. All analyses were done in duplicate or triplicate.

The chemical studies of the feces were carried out as follows :

The calcium content was determined by first boiling the sample with concentrated nitric acid and potassium permanganate. After cooling, the mixture was diluted with distilled water to 100 (or 200) $\mathrm{cc}$. volume and adjusted to the proper $\mathrm{pH}$ (4.6 to 5.2), using Brom Cresol green as indicator. The procedure then followed was the same employed for blood serum (1). (Calculation: $\frac{\text { Titer }}{2} \times 10$ equals mgm. Ca per 1 gram feces.)

TABLE II

Composition of diet employed in cases C. D. and W. G.

(Daily values)

\begin{tabular}{l|c|c}
\hline \hline & $\begin{array}{c}\text { Special } \\
\text { diet }\end{array}$ & $\begin{array}{c}\text { Standard } \\
\text { values } 70 \text { kgm. } \\
\text { adult male }\end{array}$ \\
\hline Carbohydrate & 266 & \\
Protein & 58 & \\
Fat & 163 & \\
Calories & 2763 & \\
Chloride (mgm.) & 3047 & \\
Sodium (mgm.) & 2014 & 800 \\
Calcium (mgm.) & 1140 & 1320 \\
Phosphate (mgm.) & 1300 & 12 \\
Iron (mgm.) & 7 & 5000 \\
Vitamin A (I.U.) & 6200 & 1.5 \\
Thiamin (mgm.) & 1.0 & 2.2 \\
Riboflavin (mgm.) & 1.2 to 2.4 & 75 \\
Ascorbic acid (mgm.) & 5 to 30 & \\
Vitamin D (I.U.) & 160 to 385 & \\
& & \\
\hline
\end{tabular}

2 There was no instance of diarrhea. 
For determining the chloride content, $5.0 \mathrm{cc}$. of a $0.1 \mathrm{~N}$ solution of silver nitrate were added to 2.0 gram samples of feces and the mixtures allowed to stand for several hours. Digestion then was carried out as with the calcium analysis. The mixtures were cooled, 5 per cent ferric alum added as indicator, and then titrated with a $0.1 \mathrm{~N}$ solution of potassium thiocyanate. (Calculation: $\frac{5-\text { titer } \times 3.5}{2}$ equals $\mathrm{mgm}$. chloride ion in $1.0 \mathrm{gram}$ feces.)

The phosphate in the feces was determined in Case C. D. by wet ashing of the samples with nitric and sulphuric acids and, when necessary, superoxal. The mixtures then were cooled, adjusted to known volume with distilled water, and the phosphate content measured by the Fiske-Subbarow method (2) as adapted for the Evelyn photoelectric colorimeter. In Cases A. K. and W. G., the samples of feces were ashed in a muffle furnace and the analyses then carried out as above.

In Case A. K., the sodium content of the feces was measured by the following procedure: $20 \mathrm{cc}$. of ferric sulphate and $3 \mathrm{cc}$. of concentrated sulphuric acid were added to $10 \mathrm{gram}$ samples. The mixtures were dried in an oven and then ashed in a muffle furnace. The ash was taken up in $10 \mathrm{cc}$. of distilled water and $3 \mathrm{cc}$. aliquots were analyzed for sodium by the method employed for blood (3).

The potassium content of the feces in Case A. K. was determined as follows: 2.0 gram samples of feces were dried in an oven and then ashed in a muffle furnace. The ash was taken up in $10 \mathrm{cc}$. of distilled water and $1.0 \mathrm{cc}$. aliquots were analyzed by the procedure used for blood (4).

Twenty-four hour collections of urine were obtained throughout the study. The urine was voided directly into a bottle containing toluene as a preservative and a layer of mineral oil to prevent the escape of $\mathrm{CO}_{2}$ (5). The urine was kept in an icebox and analyzed at the end of each collection for the following: $(a) \mathrm{pH}$, using the Beckman $\mathrm{pH}$ meter, (b) chloride (6), (c) calcium, (d) phosphate, and $(e)$ amino-nitrogen plus ammonium salts (7)..$^{8}$

The sodium content of the urine in Case A. $K$. was measured by the same technique employed for blood, modified as to the volume used; ferric salt was added in amounts sufficient to prevent interference by phosphates. The urinary potassium was determined as follows : $2.0 \mathrm{cc}$. samples of urine were ashed in a muffle furnace and the ash taken up in $10 \mathrm{cc}$. of distilled water; $2.0 \mathrm{cc}$. aliquots then were concentrated and analyzed by the method employed for blood.

Simultaneous studies were made of the serum electrolytes. Venous blood drawn under oil was utilized for the following analyses: chloride, $\mathrm{CO}_{2}(8), \mathrm{pH}(9)$, calcium, and phosphorus. Oxalated blood was used for the measurement of the blood urea nitrogen (10). The following

\footnotetext{
3 Referred to in the text as "ammonia" for the purpose of simplicity.
}

additional data were obtained in Case A. K. : cell volume, serum water (11), blood sodium, potassium, and total base (12), and the urea clearance (13).

After control values had been established, the effects of calcium carbonate, aluminum phosphate, and aluminum hydroxide were. studied in individual periods. A 4 to 5 day interval for adjustment to the added medication was allowed prior to the analyses. Calcium carbonate was administered in 2.0 gram amounts 10 times daily: 80 grams (400 m.eq.) were thus given in a 4-day period, containing 32 grams of calcium ion. One hundred and five cc. of aluminum phosphate were administered daily in divided doses; a total of 420 cc. (35 m.eq. HPO $)$ in a 4-day period containing, by analysis, $386 \mathrm{mgm}$. of chloride, $4368 \mathrm{mgm}$. of phosphate, and $2625 \mathrm{mgm}$. of aluminum (14). One hundred and five cc. of aluminum hydroxide were given daily in divided doses; a total of $420 \mathrm{cc}$. in a 4-day period containing $386 \mathrm{mgm}$. of chloride and 4716 $\mathrm{mgm}$. of aluminum. Complete mineral balances were not obtained since no attempt was made to measure the loss in the insensible perspiration or sweat. This loss presumably was constant, however, through the various periods in each case, since fairly constant conditions were maintained. The results are considered exclusively in relation to the individual control values.

\section{RESULTS}

The complete data are recorded in Tables III and IV.

\section{DISCUSSION}

\section{(1) Calcium carbonate}

The ingestion of 400 m.eq. daily, as calcium carbonate, was roughly accounted for by increase in total outgo, almost entirely in the stools. The increase in excretion in the urine was, for the patient A. K., only 7.3 m.eq., and for the other 2 patients, C. D. and W. G., 13.9 m.eq. and 14.1 m.eq., respectively. As was to be expected with the large addition to calcium excretion in the stools (15), there was an increase of $\mathrm{HPO}_{4}$ in the feces. For A. K. and W. G., the outgo in the stools was about double the fore-period values. These increments were, however, somewhat more than offset by a decrease in the removal of $\mathrm{HPO}_{4}$ in the urine. The increase in calcium and decrease in $\mathrm{HPO}_{4}$ excretion in the urine together markedly reduced the requirement for ammonium production as noted in Table III. Other compounds of acidbase excretion, however, are related to the change in ammonium production. For the patient A. K., a small increase in $\mathrm{Na}$ and decrease in $\mathrm{Cl}$, and a considerable reduction of $\mathrm{K}$, were found. The re- 
है

\begin{tabular}{|c|c|c|c|c|c|c|c|c|c|c|c|c|c|c|c|c|c|c|c|}
\hline & $\bar{\sigma}$ & $\stackrel{\infty}{\mathbb{N}}$ & m & $\because$ & $\overrightarrow{20}$ & $\overrightarrow{\dot{\infty}}$ & $\stackrel{N}{N}$ & $\overrightarrow{2}$ & 10 & 是 & $\overrightarrow{8}$ & 茴 & $1 \stackrel{\text { m }}{\text { q }}$ & $\stackrel{\sharp}{a}$ & $\stackrel{\infty}{\circ}$ & 送 & +9 & ț & 15 \\
\hline & 莒 & 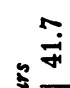 & $\stackrel{\sharp}{\sharp}$ & $\begin{array}{l}0 \\
\\
\end{array}$ & $\left|\begin{array}{c}0 \\
\text { m }\end{array}\right|$ & $\left|\begin{array}{c}\sim \\
\text { ஸे }\end{array}\right|$ & लై & $\overrightarrow{\dot{m}}$ & 1 ? & $\overrightarrow{0}$ & $\underset{+}{+}$ & $\hat{0}$ & 1 İं & $\begin{array}{l}0 \\
\text { ஜे }\end{array}$ & $\stackrel{m}{\mathbb{N}}$ & $\overrightarrow{8}$ & $+\stackrel{\text { ก }}{-1}$ & $\mid \begin{array}{l}n \\
\dot{a} \\
0\end{array}$ & $1 \stackrel{a}{0}$ \\
\hline & లో & 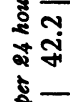 & 艧 & $\mid \begin{array}{l}0 \\
\infty \\
\infty \\
+1\end{array}$ & \begin{tabular}{|c|}
$n$ \\
0 \\
0 \\
s.
\end{tabular} \mid & \begin{tabular}{l}
$\sim$ \\
\multirow{z}{*}{}
\end{tabular} & ผ & \begin{tabular}{|c|}
\multirow{2}{*}{} \\
\end{tabular} & $+\frac{\pi}{m}$ & 苛 & \begin{tabular}{|c|}
$m$ \\
0 \\
0
\end{tabular} & बें & $\begin{array}{r}\dot{0} \\
+\stackrel{m}{\sharp} \\
\end{array}$ & 需 & ले & $\vec{\infty}$ & $1 \infty$ & 웅 & $1 \stackrel{m}{i}$ \\
\hline & $\forall$ & षैं & aे & aे & \begin{tabular}{|c|}
0 \\
0
\end{tabular} & กั่ & ชี่ & ? & $1 \stackrel{+}{0}$ & $\stackrel{\sim}{=}$ & & & & & & & & & \\
\hline & $\ddot{\varpi}$ & $\begin{array}{l}\infty \\
\text { ஜn }\end{array}$ & वें & 帝 & م்ْ & 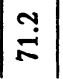 & กั. & $\begin{array}{l}0 \\
8 \\
8\end{array}$ & $+\hat{i}$ & $\mid \begin{array}{l}\mathfrak{s} \\
\end{array}$ & & & & & & & & & \\
\hline & $\overline{0}$ & $\stackrel{\circ}{i}$ & i̊ & $\stackrel{i}{i}$ & $\exists$ & ले & $\stackrel{\circ}{+}$ & $\stackrel{0}{+}$ & $+\stackrel{0}{\mathrm{~N}}$ & $\hat{i}$ & $\stackrel{n}{i}$ & $\stackrel{\hat{i}}{\hat{i}}$ & $+\stackrel{+}{0}$ & $\stackrel{m}{+}$ & $\stackrel{\circ}{+}$ & $\stackrel{n}{q}$ & $+\stackrel{0}{i}$ & $\stackrel{a}{m}$ & $+\stackrel{+}{-}$ \\
\hline & : & 铝 & ò & $\tilde{0}$ & जे & $\mid$\begin{tabular}{l}
$\infty$ \\
\multirow{i}{*}{}
\end{tabular} & ח̊ & $\mid \begin{array}{l}\infty \\
i \\
i\end{array}$ & 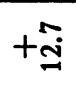 & $\hat{a}$ & 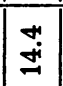 & $\stackrel{\infty}{=}$ & $+\stackrel{m}{m}$ & $\vec{z}$ & $\tilde{q}$ & $\frac{9}{7}$ & $+\stackrel{n}{n}$ & 芦 & $+\infty$ \\
\hline & లే & to & $\stackrel{m}{7}$ & $\mid \begin{array}{l}0 \\
0 \\
0\end{array}$ & $\left|\begin{array}{l}0 \\
0 \\
0 \\
\text { ల్ }\end{array}\right|$ & $\left|\begin{array}{c|}0 \\
\dot{+} \\
\text { d. }\end{array}\right|$ & $\underset{\infty}{\infty}$ & $\left|\begin{array}{l}0 \\
0 \\
0 \\
0\end{array}\right|$ & +离 & \begin{tabular}{|l|}
$\infty$ \\
$\infty$ \\
$\infty$ \\
$\infty$
\end{tabular} & Hु̉ & 㝘 & $+\hat{\overparen{\jmath}}$ & mo & $\stackrel{+}{\Delta}$ & $\mid \begin{array}{l}a \\
\dot{\alpha}\end{array}$ & 1 iे & $\frac{9}{\text { in }}$ & $1 \stackrel{+}{N}$ \\
\hline & 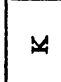 & & $\begin{array}{l}0 \\
0\end{array}$ & $\begin{array}{l}0 \\
0 \\
0\end{array}$ & $\stackrel{9}{=}$ & $\vec{\Xi}$ & 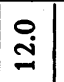 & $\stackrel{0}{i}$ & $+\stackrel{+}{n}$ & 号 & & & & & & & & & \\
\hline & $\ddot{z}$ & $\stackrel{m}{-}$ & aे & $\exists$ & $\Xi$ & 9 & $\hat{0}$ & 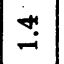 & $+\stackrel{n}{0}$ & $\stackrel{\circ}{i}$ & & & & & & & & & \\
\hline \multirow{7}{*}{ 苋 } & $\bar{\sigma}$ & $\stackrel{\infty}{\mathbb{N}}$ & $\underset{\infty}{\infty}$ & $\mid \begin{array}{l}0 \\
\end{array}$ & $|\stackrel{0}{\underset{1}{1}}|$ & $\frac{\Upsilon}{\infty}$ & กั & $\left|\begin{array}{l}\infty \\
\multirow{+}{*}{}\end{array}\right|$ & $1 \stackrel{\infty}{\sim}$ & ஸุ' & $\left|\begin{array}{l}0 \\
0 \\
0\end{array}\right|$ & $\stackrel{\tilde{N}}{\stackrel{n}{*}}$ & $1 \stackrel{9}{\stackrel{g}{\xi}}$ & $\underset{\infty}{\vec{\infty}}$ & : & $\mid \frac{0}{\infty}$ & + & $\begin{array}{l}n \\
8 \\
8\end{array}$ & $1 \overline{0}$ \\
\hline & 芯 & $\frac{\text { บ }}{m}$ & n̊. & 商 & $\overrightarrow{\mathbf{I}}$ & 苛 & $\tilde{I}$ & $\Xi$ & $1 \stackrel{2}{2}$ & 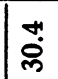 & $\overrightarrow{0}$ & $\underset{\infty}{\infty}$ & $1 \stackrel{\infty}{=}$ & $\stackrel{n}{*}$ & வ̊ & : & 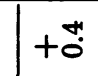 & $\stackrel{?}{\sim}$ & $1 \tilde{\text { ș }}$ \\
\hline & లో & $\stackrel{\circ}{=}$ & $\stackrel{0}{\stackrel{0}{3}}$ & $\vec{\Xi}$ & مْ & ก̃ & $\stackrel{n}{=}$ & $\ddot{a}$ & $+\stackrel{?}{n}$ & $\stackrel{0}{\infty}$ & 首 & à & $+\stackrel{a}{\stackrel{m}{-}}$ & นa & $\stackrel{0}{\infty}$ & ஸ̃ & $1 \stackrel{\infty}{i}$ & $\overline{\underline{I}}$ & $+\overrightarrow{0}$ \\
\hline & 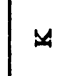 & & $\ddot{\tilde{\theta}}$ & $\ddot{\tilde{\theta}}$ & $\vec{g}$ & กิ่ & ஸั & ñ & $1 \stackrel{\infty}{\underset{I}{~}}$ & $\begin{array}{l}0 \\
8\end{array}$ & & & & & & & & & \\
\hline & $\ddot{z}$ & : & 亩 & นึ่ & $\begin{array}{l}\infty \\
\infty \\
\infty \\
\infty\end{array}$ & å. & \%̊ำ & ชุ: & $+\stackrel{n}{n}$ & no & & & & & & & & & \\
\hline & 要 & सें & $\begin{array}{l}0 \\
\dot{m}\end{array}$ & 离 & ْ̊ & $\stackrel{\text { i }}{\text { i }}$ & ชี & $\begin{array}{l}0 \\
\text { î. }\end{array}$ & $1 \stackrel{+}{\underset{乛}{*}}$ & 官 & 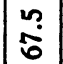 & 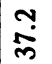 & I & $\underset{\infty}{+}$ & $\stackrel{1}{n}$ & ! & $+\stackrel{m}{n}$ & $\begin{array}{l}\infty \\
0 \\
\dot{0} \\
\end{array}$ & 1 i্ं \\
\hline & 咭 & $\frac{0}{6}$ & సิ & స్ & $\mid \begin{array}{l}0 \\
0 \\
0 \\
0\end{array}$ & $\frac{n}{6}$ & 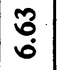 & $\begin{array}{l}2 \\
0 \\
0 \\
0\end{array}$ & $+\frac{\pi}{0}$ & ๙ू. & $\mid \begin{array}{l}0 \\
\text { in } \\
\text { in }\end{array}$ & 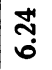 & $+\stackrel{\infty}{+}$ & พั & $\stackrel{?}{\rightarrow}$ & พี & 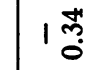 & ầ & $+\frac{7}{0}$ \\
\hline \multicolumn{2}{|c|}{ 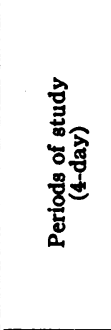 } & 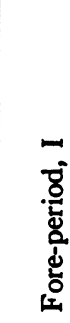 & 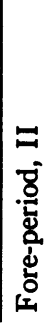 & 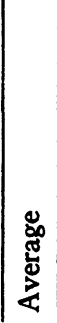 & 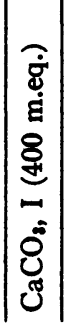 & $\begin{array}{l}\square \\
0 \\
0 \\
0 \\
0\end{array} \mid$ & 吾 & 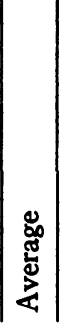 & $\begin{array}{l}\text { : } \\
\text { J్ } \\
\text { త్ }\end{array}$ & 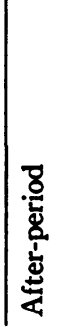 & 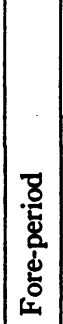 & 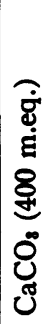 & 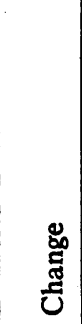 & 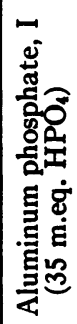 & 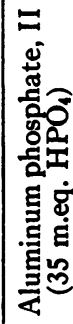 & 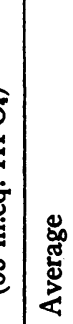 & 总 & 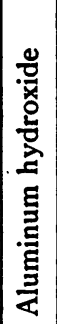 & 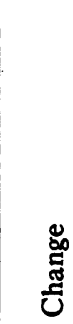 \\
\hline & 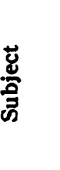 & \multicolumn{9}{|c|}{ 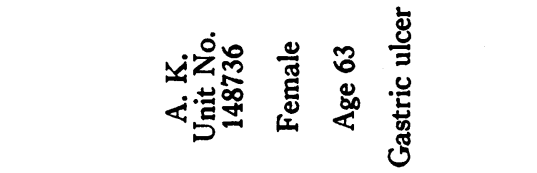 } & \multicolumn{4}{|c|}{ 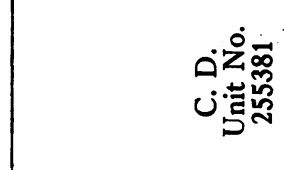 } & \multicolumn{4}{|c|}{ 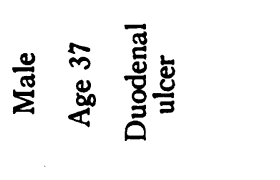 } & \\
\hline
\end{tabular}




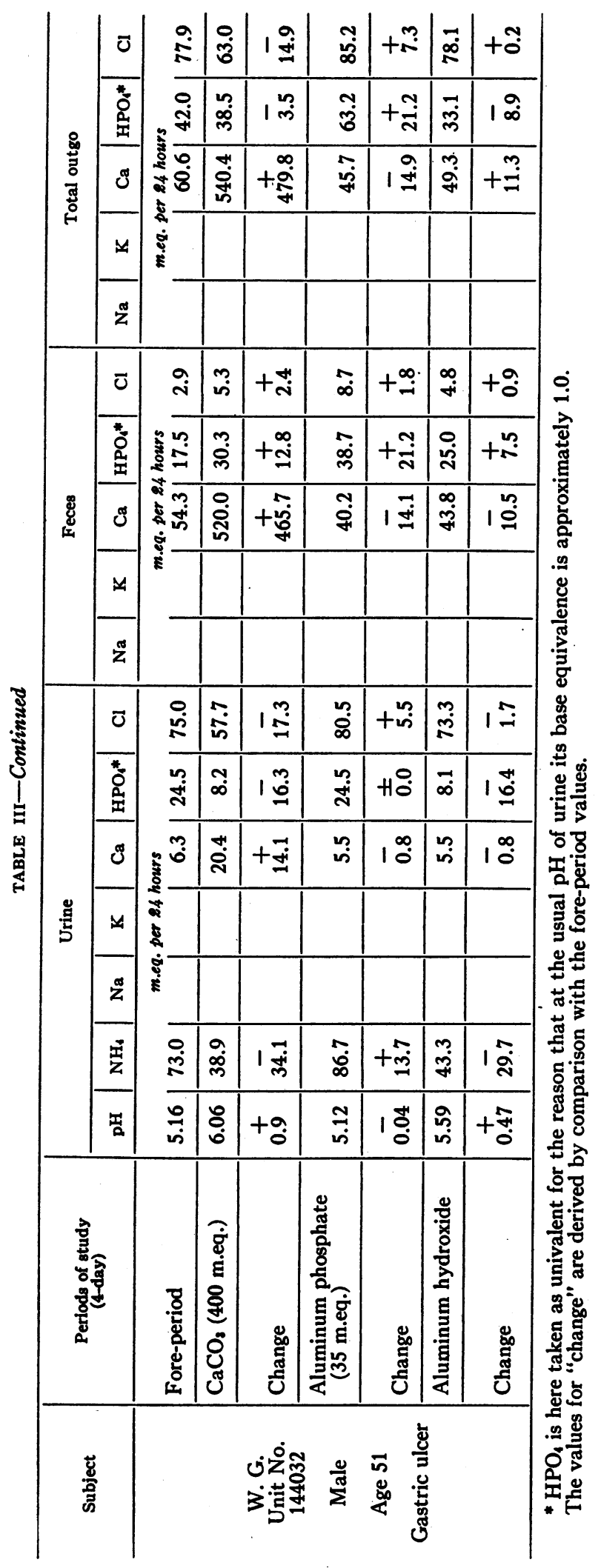


EFFECT OF ANTI-ACID AGENTS ON MINERAL EXCRETION

TABLE IV

Effect of calcium carbonate, aluminum phosphate, and aluminum hydroxide on various constituents of the blood plasma

\begin{tabular}{|c|c|c|c|c|c|c|c|c|c|c|c|c|c|}
\hline Subject & $\begin{array}{c}\text { Periods of study } \\
\text { (4-day) }\end{array}$ & $\mathrm{Cl}$ & $\mathrm{CO}_{2}$ & $\mathrm{pH}$ & $\mathbf{P}$ & $\mathbf{C a}$ & $\mathbf{K}$ & $\mathbf{N a}$ & $\begin{array}{l}\text { Total } \\
\text { base }\end{array}$ & BuN & $\begin{array}{c}\text { Urea } \\
\text { clearance }\end{array}$ & $\begin{array}{c}\text { Cell } \\
\text { volume }\end{array}$ & $\begin{array}{c}\text { Scrum } \\
\text { water }\end{array}$ \\
\hline \multirow{6}{*}{$\begin{array}{l}\text { A. K. } \\
\text { Unit No. } \\
148736 \\
\text { Female } \\
\text { Age } 63 \\
\text { Gastric } \\
\text { ulcer }\end{array}$} & Fore-period I & \multicolumn{2}{|c|}{$m M . / L$} & 7.43 & $\begin{array}{c}m M . / L . \\
1.26\end{array}$ & 4.9 & \multicolumn{2}{|c|}{\begin{tabular}{c|c|}
\multicolumn{2}{c|}{$m$. eq. $/ L}$. \\
5.30 & 143.0
\end{tabular}} & 155.3 & $\begin{array}{c}\underset{\text { per cent }}{m g m .} \\
15.4\end{array}$ & $\begin{array}{c}\text { per cent } \\
\text { average } \\
\text { normal } \\
66\end{array}$ & $\begin{array}{c}\text { per cent } \\
40.4 \\
\end{array}$ & $\begin{array}{c}\begin{array}{c}\text { grams } \\
\text { per cent }\end{array} \\
91.1 \\
\end{array}$ \\
\hline & Fore-period II & 102.2 & 31.8 & 7.47 & 1.29 & 4.7 & 5.20 & 144.3 & 153.3 & 14.6 & 81 & 37.0 & 90.97 \\
\hline & $\begin{array}{l}\text { Calcium } \\
\text { carbonate I }\end{array}$ & 100.7 & 31.7 & 7.50 & 1.35 & 5.75 & 5.20 & 147.9 & 160.0 & 17.0 & 44 & 41.0 & 90.42 \\
\hline & $\begin{array}{l}\text { Calcium } \\
\text { carbonate II }\end{array}$ & 100.5 & 31.9 & 7.44 & 1.19 & 5.35 & 5.00 & 145.2 & 158.5 & 17.8 & 65 & 41.5 & 90.68 \\
\hline & $\begin{array}{l}\text { Calcium } \\
\text { carbonate III }\end{array}$ & 103.1 & 29.7 & 7.47 & 1.22 & 5.1 & 5.30 & 145.0 & 156.1 & 15.5 & 65 & 37.5 & 91.14 \\
\hline & After-period & 100.2 & 30.9 & 7.43 & 1.13 & 5.1 & 5.30 & 143.8 & & 13.2 & 76 & 37.2 & 91.06 \\
\hline \multirow{2}{*}{$\begin{array}{c}\text { C. D. } \\
\text { Unit No. } \\
255381\end{array}$} & Fore-period & 101.7 & 29.6 & 7.46 & 1.32 & 5.05 & & & & 9.4 & & & \\
\hline & $\begin{array}{l}\text { Calcium } \\
\text { carbonate }\end{array}$ & 99.6 & 29.6 & 7.46 & 0.97 & 5.15 & & & & 13.5 & & & \\
\hline \multirow{3}{*}{$\begin{array}{c}\text { Male } \\
\text { Age } 37 \\
\begin{array}{c}\text { Duodenal } \\
\text { ulcer }\end{array}\end{array}$} & $\begin{array}{l}\text { Aluminum } \\
\text { phosphate I }\end{array}$ & 103.4 & 28.4 & 7.48 & 1.0 & 5.7 & & & & 12.0 & & & \\
\hline & $\begin{array}{l}\text { Aluminum } \\
\text { phosphate II }\end{array}$ & 100.5 & 29.8 & 7.46 & 1.22 & 5.5 & & & & 10.0 & & & \\
\hline & $\begin{array}{l}\text { Aluminum } \\
\text { hydroxide }\end{array}$ & 102.4 & 28.5 & 7.47 & 1.13 & 5.25 & & & & 15.4 & & & \\
\hline \multirow{2}{*}{$\begin{array}{c}\text { W. G. } \\
\text { Unit No. } \\
144032\end{array}$} & Fore-period & 96.7 & 28.6 & 7.43 & 1.42 & 4.65 & & & & 14.2 & & & \\
\hline & $\begin{array}{l}\text { Calcium } \\
\text { carbonate }\end{array}$ & 95.6 & 29.9 & 7.43 & 1.35 & 5.1 & & & & 13.8 & & & \\
\hline $\begin{array}{c}\text { Male } \\
\text { Age 51 }\end{array}$ & $\begin{array}{l}\text { Aluminum } \\
\text { phosphate }\end{array}$ & 106.9 & 28.2 & 7.42 & 1.40 & 4.75 & & & & 16.3 & & & \\
\hline $\begin{array}{r}\text { Gastric } \\
\text { ulcer }\end{array}$ & $\begin{array}{l}\text { Aluminum } \\
\text { hydroxide }\end{array}$ & 101.6 & 27.4 & 7.41 & 1.40 & 5.05 & & & & 14.0 & & & \\
\hline
\end{tabular}

maining components, $\mathrm{Mg}, \mathrm{SO}_{4}$, and organic acids were not measured. The accuracy of ammonium adjustment is shown by the slight extent of change in urine $\mathrm{pH}$. For the patient W. G., a considerable, and for C. D., a much larger, reduction of chloride in the urine was found. Excretion of this anion in the urine is known to fluctuate widely even in the presence of a constant intake $(16,17)$.

The changes in mineral excretion induced by calcium carbonate occurred within 24 hours after the addition of the alkali to the regimen. Mineral excretion returned to original levels almost equally soon after the discontinuation of calcium carbonate therapy.

The use of calcium carbonate did not alter the electrolyte constitution of the blood plasma ( $\mathrm{Ta}$ ble IV).
(2) Aluminum phosphate

The ingestion of 35 m.eq. $\mathrm{HPO}_{4}$ as aluminum phosphate caused a roughly equivalent increase in the stools and had no appreciable effect on $\mathrm{HPO}_{4}$ output in the urine. Calcium excretion in the stools was to a slight extent reduced. There was no appreciable change in the calcium output in the urine. Some increase in chloride and, also, ammonium excretion in the urine was found. A relationship of these changes to phosphate ingestion was not apparent. No alterations were noted in the electrolyte components of the blood plasma.

\section{(3) Aluminum hydroxide}

Aluminum hydroxide caused a considerable increase in $\mathrm{HPO}_{4}$ excretion in the stools, as demon- 
strated by the data from the two periods of study. This finding is in agreement with the results obtained by other workers (18). A roughly equivalent decrease of the excretion of $\mathrm{HPO}_{4}$ in the urine was found and, along with it, the to be expected reduction of ammonium. For patient C. D., this was exactly equivalent to the decrease of $\mathrm{HPO}_{4}$. The total outgo of $\mathrm{HPO}_{4}$ was not increased. Aluminum hydroxide did not alter the electrolytes of the blood plasma, thus confirming previous observations (19).

\section{CONCLUSION}

The ingestion of calcium carbonate, aluminum phosphate, or aluminum hydroxide, in the quantities used in the treatment of peptic ulcer, places no appreciable burden on the processes of acidbase metabolism. The necessary adjustments of acid-base excretion are relatively small and are accomplished with a remarkable precision.

The electrolyte constitution of the blood plasma is not disturbed.

The author gratefully acknowledges the generous aid given by Dr. James L. Gamble in the preparation of the paper and the valuable technical assistance of Miss Jacqueline S. Front.

\section{BIBLIOGRAPHY}

1. Clarke, E. P., and Collip, J. C., A study of the Tisdall method for the determination of blood serum calcium with a suggested modification. J. Biol. Chem., $1925,63,461$.

2. Fiske, C. H., and Subbarow, Y., The colorimetric determination of phophorus. J. Biol. Chem., 1925, 66, 375. Applied to Evelyn Photoelectric Colorimeter, cf. Bulletin No. 460, Rubicon Co., Philadelphia, $\mathrm{Pa}$.

3. Butler, A. M., and Tuthill, E., An application of the uranyl zinc acetate method for the determination of sodium in biological material. J. Biol. Chem., 1931, 93, 171.

4. Shohl, A. T., and Bennett, H. B., A micro method for the determination of potassium as iodoplatinate. J. Biol. Chem., 1928, 78, 643 . Modified by Eichelberger.

5. Marshall, E. K., Jr., The effect of loss of carbon dioxide on the hydrogen-ion concentration of the urine. J. Biol. Chem., 1922, 51, 3.

6. Wilson, D. W., and Ball, E. G., A study of the estimation of chloride in blood and serum. J. Biol. Chem., 1928, 79, 221.

7. Henriques, V., and Sörensen, S. P. L., Ueber die quantitative Bestimmung der Aminosäuren, Poly- peptide und der Hippursäure im Harne durch Formoltitration. Ztschrf. f. Physiol. Chem., 1909, 63, 27.

8. Van Slyke, D. D., and Neil, J. M., The determination of gases in blood and other solutions by vacuum extraction and manometric measurement. J. Biol. Chem., 1924, 61, 523.

9. Hastings, A. B., Sendroy, J., Jr., and Robson, W., The colorimetric determination of the $\mathrm{pH}$ of the urine. J. Biol. Chem., 1925, 65, 381.

10. Van Slyke, D. D., Determination of urea by gasometric measurement of the carbon dioxide formed by the action of urease. J. Biol. Chem., 1927, 73, 695.

11. Hastings, A. B., and Eichelberger, L., The exchange of salt and water between muscle and blood. I. The effect of an increase in total body water produced by the intravenous injection of isotonic salt solutions. J. Biol. Chem., 1937, 117, 73.

12. Stadie, W. C., and Ross, E. C., A micro method for the determination of base in blood, serum and other biological material. J. Biol. Chem., 1925, 65, 735.

13. Van Slyke, D. D., and others, Observations on the courses of different types of Bright's disease and on resultant changes in renal anatomy. Medicine, 1930, 9, 257.

14. Front, J., and Kirsner, J. B., An improved method for the determination of aluminum in biological material using the Evelyn photoelectric colorimeter. J. Lab. and Clin. Med., 1942, 27, 1598.

15. a. Forbes, E. B., and Keith, M. H., A review of the literature of phosphorus compounds in animal metabolism. Ohio Agricultural Exp. Station, Technical Series Bulletin No. 5, Wooster, Ohio, 1914.

b. Telfer, S. V., Studies on calcium and phosphorus metabolism. I. The excretion of calcium and phosphorus. Quart. J. Med., 1922, 16, 45.

16. Gamble, J. L., Chemical Anatomy, Physiology and Pathology of Extracellular Fluid. A Lecture Syllabus. Dept. Pediatrics, Harvard Medical School, Boston, 1939.

17. Atchley, D. W., Loeb, R. F., et al., On diabetic acidosis. J. Clin. Invest., 1933, 12, 297.

18. a. Fauley, G. B., Ivy, A. C., Terry L., and Bradley, W. B., An attempt to prevent post operative jejunal ulcer by aluminum hydroxide therapy. Am. J. Digest. Dis., 1939, 5, 792.

b. Fauley, G. B., Freeman, S., and Ivy, A. C., Aluminum phosphate in the therapy of peptic ulcer. Arch. Int. Med., 1941, 67, 563.

c. Freeman, S., and Freeman, W. M. C., Phosphorus retention in children with chronic renal insufficiency. Am. J. Dis. Child., 1941, 61, 981.

19. Kirsner, J. B., The effect of aluminum hydroxide on the acid-base balance and on renal function. Am. J. Digest. Dis., 1941, 8, 160. 